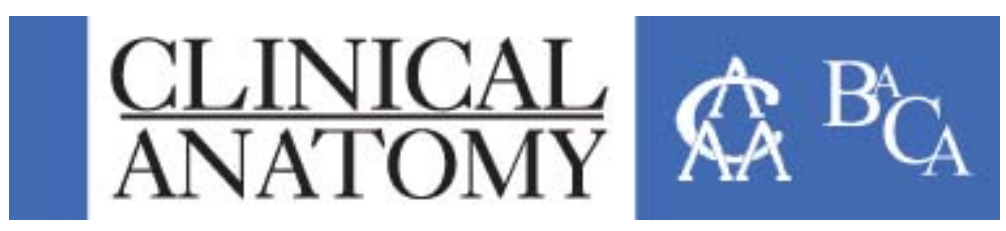

\title{
THE INNOMINATE TUBERCLE OF THE FEMUR IS A CONSISTENT SURGICAL LANDMARK AND SHOWS NO VARIATION BETWEEN SEX AND SIDE: AN OSTEOLOGICAL STUDY
}

\begin{tabular}{|r|l|}
\hline Journal: & Clinical Anatomy \\
\hline Manuscript ID & CA-20-0337.R2 \\
\hline Wiley - Manuscript type: & Original Communications \\
\hline Keywords: & anatomy, surgery \\
\hline Additional Keywords: & $\begin{array}{l}\text { arthroplasty, direct anterior approach, innominate tubercle, femoral } \\
\text { osteotomy, femoral neck, femur }\end{array}$ \\
\hline \multicolumn{2}{|l}{} \\
\hline
\end{tabular}

\section{SCHOLARONE ${ }^{m}$ \\ Manuscripts}


THE INNOMINATE TUBERCLE OF THE FEMUR IS A CONSISTENT SURGICAL LANDMARK AND SHOWS NO VARIATION BETWEEN SEX AND SIDE: AN OSTEOLOGICAL STUDY

\section{ABSTRACT}

\section{Introduction}

During direct anterior approach hip arthroplasty, the innominate tubercle (IT) of the femur is used as a reference point for femoral neck osteotomy. A recent study found that IT dimensions are unaffected by femoral length, neck-shaft angle, and neck length. This study investigated the position of the tubercle relative to femoral side, sex and femoral neck dimensions.

\section{Materials and Methods}

One hundred and ninety femora (ninety-five pairs, complete with biographical data) from St. Bride's Church, London, were photographed with the apex of the tubercle marked. Using imaging software, axes were superimposed onto the image, allowing the $X$ and $Y$ co-ordinates of the tubercle apex to be derived. Statistical analysis was carried out.

\section{Results}

The position of the tubercle did not vary significantly and did not correlate with femoral side $(P>0.05)$. $P$ values for variation of the $X$ and $Y$ coordinates were 0.351 and 0.996 in left and right femora, respectively. The tubercle position did not vary significantly with sex $(P>0.05)$. $P$ values for variation of the $\mathrm{X}$ and $\mathrm{Y}$ coordinates were 0.254 and 0.634 in males and females respectively. There was little variation between tubercle position and femoral neck dimensions: correlation coefficient -0.20 ( $\mathrm{x}$ coordinate) and -0.12 (y coordinate).

\section{Conclusions}

The data showed that the position of the IT did not vary significantly with either sex or side and that, therefore, the tubercle can be considered a reliable surgical landmark during arthroplasty surgery. Keywords: arthroplasty; femur; femur neck; innominate tubercle; direct anterior approach; femoral osteotomy 


\section{INTRODUCTION}

One of the earliest mentions of the innominate tubercle (IT) was the 1918 edition of Gray's Anatomy, which stated: 'A prominence, of variable size, occurs at the junction of the upper part of the neck with the greater trochanter, and is called the tubercle of the femur' (Gray, 1918). This landmark has also been referred to in the literature as: the cervical tubercle, the superior cervical tubercle, and the femoral tubercle (Frazer, 1920; Prasad et al., 1996; O'Rahilly et al., 2004); however, it is most commonly referred to as the "innominate tubercle" of the femur. Limited research has been conducted into this landmark, which may account for the absence of a universal name. Currently, we know that the tubercle is positioned on the anterior aspect of the proximal femur, palpable on the intertrochanteric line, and that both the iliofemoral ligament and part of the acetabular capsule attach here (Standring, 2008) (Fig. 1).

Figure 1.

Several hip approaches are available to the surgeon to allow implantation. The direct anterior approach (DAA) has increased in popularity amongst arthroplasty surgeons, due to a desire for less invasive, tissue-sparing procedures with faster recovery times, decrease in dislocation, and for patients to incur less pain postoperatively (Connolly and Kamath, 2016). Williams et al. (2015) emphasise the importance of this tubercle during DAA surgery, describing it as a "lighthouse" for femoral osteotomy. It has been estimated that $25 \%$ of arthroplasty surgeons choose to use the DAA method for hip arthroplasty (Keggi, 2014). Although this landmark is relied upon intraoperatively, there is little evidence in the literature to show that it is in fact a reliable and consistent reference point between subjects and sides. The aim of this novel study was to provide osteological evidence, from a large sample, on whether the innominate tubercle is a reliable and dependable reference point for DAA hip arthroplasties. 


\section{MATERIALS AND METHODS}

This study used an archaeological skeletal population of 227 paired femora of European origin, with known age, date of death and sex, from the crypt of St. Bride's Church, London. St Bride's crypt individuals are a significant and large biographical skeletal collection. The skeletal remains from St Bride's crypt provide an important insight to a post-medieval population in London and their welldocumented background, including age and date of death, socioeconomic status, demographics, cause of death and occupation, makes this population ideal for comparative studies between osteological assemblages (Thomas et al., 2018). Using paired femora enables comparisons to be made between the left and right tubercle in the same individual. The biographical data, accessed via the Wellcome Osteological Research Database (WORD) (Museum of London, 2018), allowed us to identify variations due to sex, age or pathology.

Ninety-five pairs of femora were suitable for use in this study. Exclusion criteria were absence of, or gross damage to, the proximal third of the femur, if it rendered it impossible to identify the tubercle in either of the pair. The sex of two pairs of femora was unrecorded, and these were excluded from male/female analysis.

\section{Measurements taken}

To eradicate any inter-observer variability, the IT was marked on each femur by one operator only, and all measurements were taken by this same operator. On each femur, the tubercle was first palpated at the junction of the greater trochanter with the neck of the femur, and the apex was then marked using a small piece of "white tac". The white tac was necessary to facilitate identification of the apex of the tubercle on the image. The proximal third of each femur, with the tubercle marked, was photographed using a Nikon 3100 DSLR camera, mounted on a camera stand. The images were uploaded to a computer. Analysis was carried out using the software PhotoScape X (version 2.7.1). Fahmy et al. (2018) trialled a similar method for plotting the IT on a Cartesian axis from two 
dimensional (2D) images. In addition, Anakwenze et al. (2014) used an identical method for plotting the supinator tubercle of the ulna, using three-dimensional coordinates from axes drawn on a computed tomography (CT) image. For this study, axes were drawn onto each image, using anatomical landmarks to ensure that the axes were standardised between images. The $y$ axis was first defined as a line transecting the femoral neck at its narrowest point; the image was then rotated until the $y$ axis was vertical, using gridlines for reference. An $\mathrm{x}$ axis was then plotted at the midpoint of, and perpendicular to, the $y$ axis, extending from the end of the femoral head to the farthest lateral point of the greater trochanter. The apex of the innominate tubercle was marked, and the coordinates of the tubercle apex were then derived using the software (Fig. 2). The $x$ and $y$ coordinates were then expressed as a percentage of the length of the axes. The data were recorded on Microsoft Excel (version 15.31, Microsoft Corporation) to create a contour plot. Prior to investigating the femora from St. Brides, a random left and right femur from St George's University of London was used to trial the method and to assess intra-observer variation and reliability. The IT apex was marked and photographed on five separate occasions by the same operator on both the left and right femur. Five sets of calculations were then done for position of the tubercle as a percentage of axis length on both left and right femora and intra-rater reliability was measured.

Figure 2

Statistical Analysis

The IBM SPSS statistical software package (version 25.0, IBM, Armonk, New York) was used for all statistical analyses. Statistical differences and relationships between groups were examined using the unpaired Student's $t$-test for comparison of sex or independent Student's $t$-test for side comparison. Correlations were tested using Pearson correlation coefficient (r). Coordinate data were used to produce contour plots for males, females, and males and females combined. Intra-rater 
reliability was analysed using co-efficient of variance. All values have been expressed as mean \pm standard deviation of the mean. A $p$ value of $<0.05$ was considered significant. 
RESULTS

Of the 227 skeletons in the crypt of St. Bride's Church, 95 had paired femora where the innominate tubercle could be palpated and clearly marked (Table 1); the rest had severe post-mortem damage to the femora, or they were absent. Therefore, in total, data were collected from 190 individual femora, or 95 pairs.

\begin{tabular}{|c|c|c|c|}
\hline & Male & Female & Unknown \\
\hline Left & $42(44.2 \%)$ & $51(53.7 \%)$ & $2(2.1 \%)$ \\
\hline Right & $42(44.2 \%)$ & $51(53.7 \%)$ & $2(2.1 \%)$ \\
\hline
\end{tabular}

Table 1. Demographics of St. Bride's femora (Museum of London, 2018)

Coordinate data were used to produce contour plots for males (Fig. 3a), females (Fig. 3b), and males and females combined (Fig. 3c)

Figure $3 a$

Figure $3 b$

Figure $3 c$

t-Test Results

In each case, the $P$ value was $>0.05$, showing that there was no significant variation in the position of the IT with regards to sex, side or femoral neck dimensions (Fig. 4a-b, Table 2). 


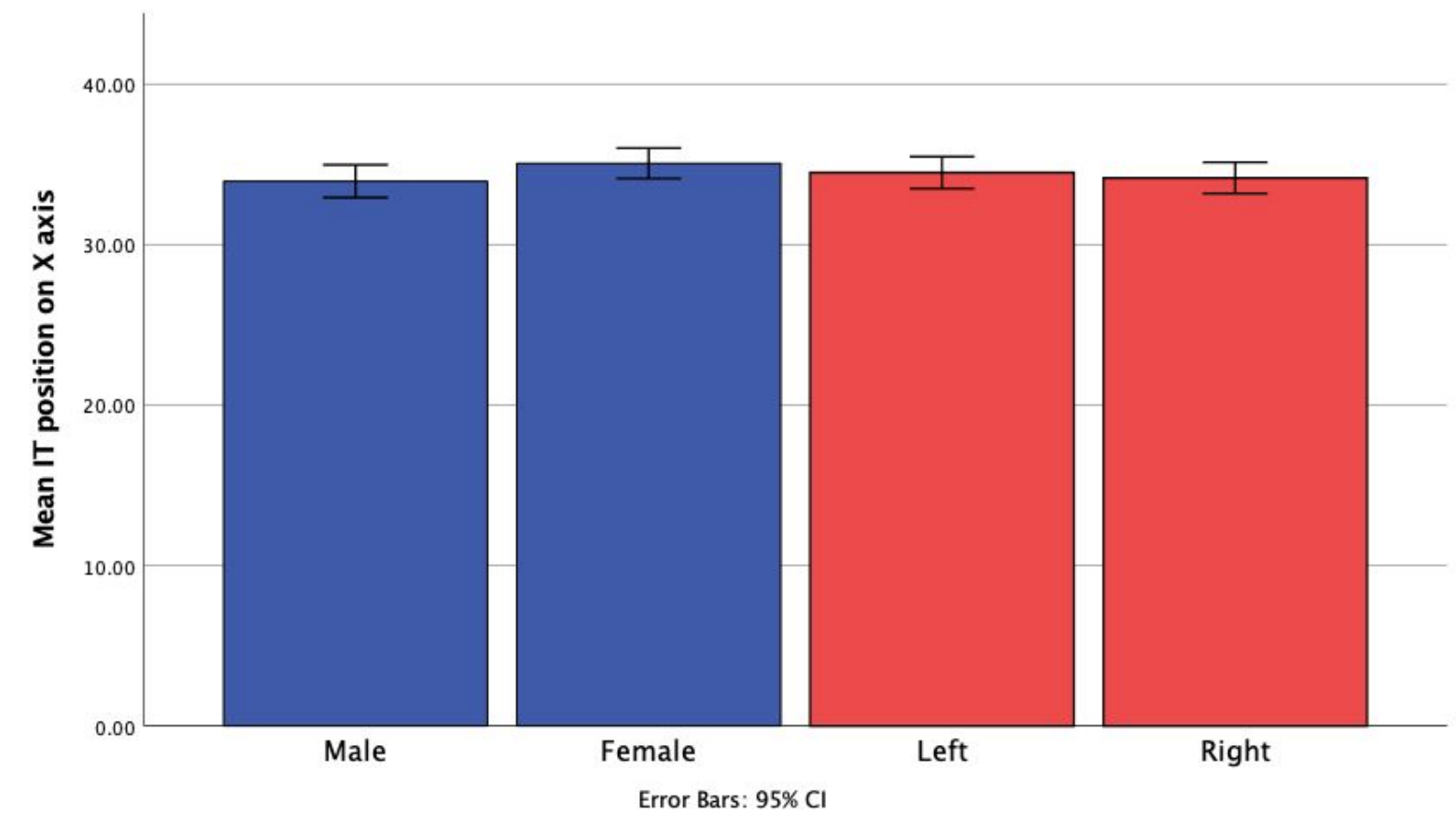

Figure 4a: IT position on $\mathrm{X}$ axis with respect to sex and side

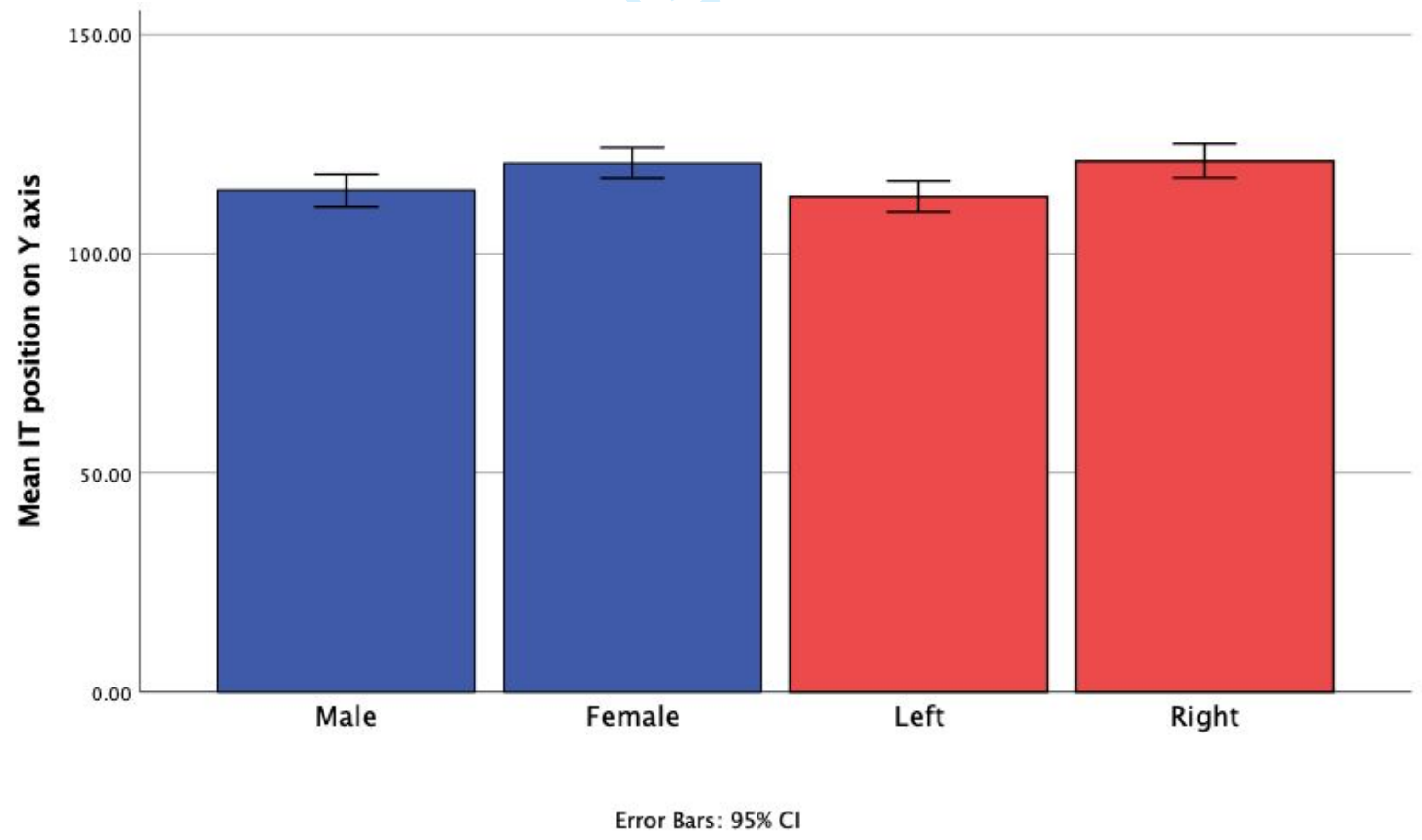

Figure 4b: IT position on $\mathrm{Y}$ axis with respect to sex and side 


\begin{tabular}{|l|l|l|}
\hline Comparisons & T Value & P Value \\
\hline$X$ coordinate in left and right femora & 0.491 & 0.351 \\
\hline$Y$ coordinate in left and right femora & -0.453 & 0.966 \\
\hline$X$ coordinate in males and females & -1.123 & 0.226 \\
\hline$Y$ coordinate in males and females & -2.160 & 0.856 \\
\hline
\end{tabular}

Table 2. Summary of data for t-Test

Correlation coefficient

The correlation coefficients of tubercle position and femoral neck dimensions were -0.20 ( $\mathrm{x}$ coordinate) and -0.12 (y coordinate). Correlation coefficients for females were -0.13 and -0.21 for the $x$ and $y$ coordinates, respectively and -0.18 ( $x$ coordinate) and -0.09 ( $y$ coordinate) for males. This was also the case for IT position with respect to side; the correlation coefficient was -0.13 ( $x$ coordinate) and -0.32 ( $y$ coordinate) in left femora and -0.21 ( $x$ coordinate) and -0.11 (y coordinate) in right femora.

Intra-rater reliability test

The coefficient of variance for tubercle position on the $\mathrm{x}$ axis was 0.13 and 0.96 in left and right femora, respectively. For tubercle position on the y axis, the coefficient of variance was 0.01 and 0.16 in left and right femora, respectively. All values were $<1$, indicating that variance was low, and the readings were reliable. 


\section{DISCUSSION}

In this large osteology study, we report that the position of the IT does not vary significantly with femoral side or sex. Similarly, there was little variation between tubercle position and femoral neck dimensions. This study indicates that the IT occupies a consistent position on the femoral neck. This once again reinforces the IT as an adequate surgical landmark which can be reliably used during hip surgery, particularly arthroplasty. To our knowledge, only one previous study has investigated the reliability of the IT in surgery. Williams et al. (2014) conducted a study on a series of 151 surgical patients to evaluate the effectiveness of using the innominate tubercle as a lateral reference point for femoral neck osteotomy during DAA arthroplasty. Leg length discrepancy was used as a surrogate marker to measure accuracy of the osteotomy. Results from this study showed a leg length discrepancy range of -3 to $+3 \mathrm{~mm}$, with a mean of $+1.7 \mathrm{~mm}$. It was thus concluded that the innominate tubercle was a reliable reference point (Williams et al., 2014).

Prasad et al. (1996) measured the prominence of the tubercle in 171 unpaired femora of Indian origin. The sex of the femora was estimated by the authors from femoral head dimensions. To measure protrusion of the innominate tubercle or "superior cervical tubercle" as Prasad et al. (1996) called it, with relation to side, a scoring system of 0 (absent) to 4 (highly prominent) was used; they concluded that there was no significant difference between prominence, sex, and side. Their study was limited by the femora being unpaired, and of unknown age and sex. Kielbasinski Podlaszewska et al. (2017) improved on this by using 79 pairs of adult (defined as over 18 years) femora, with records of age, sex and date of death, to assess the variability of IT dimensions. The results of their study support Prasad et al.'s (1996), conclusion that there was no significant association between prominence and sex $(P>0.05)$, and that the length, width, and height of the tubercle did not vary significantly with sex. 
In our study, in order to allow for variations of the femoral neck between individuals, the y-coordinate was plotted using an anatomical feature (the narrowest part of the femoral neck) as a reference point. The geometry of the proximal femur is known to vary with sex and ancestry (Meeusen et al., 2015), so it was important to use a standardised method that would not be affected by variations in the geometry of the femoral neck. It was found that the position of the IT did not change significantly with respect to the $\mathrm{x}$ or $\mathrm{y}$ axis lengths, and therefore, with dimensions of the femoral neck. Although it appeared to vary more in the $y$ axis than the $x$ axis, the Pearson correlation coefficient in both cases was less than 0.3 , suggesting that there was "negligible correlation" (Mukaka, 2012) between the two variables.

Similarly, the position of the IT on the $\mathrm{x}$ or $\mathrm{y}$ axis did not vary in relation to side of the body (Table 2). This is in keeping with findings from both Prasad et al. (1996) and Kielbasinski Podlaszewska et al. (2017), that the prominence of the IT does not vary with femoral side. It is known that muscle tension results in morphological changes, such as an increase in tubercle size (Chamay and Tschantz, 1971; Knüsel C, 2000; Makhni et al., 2014), and that it is common to see more prominent sites of muscle attachments (Genge, 2004) and slight bone lengthening (of upper limbs) (Glassman and Dana, 1992; Kanchan et al., 2007) on the dominant side. It may, then, be surprising that there was no significant variation in the IT between left and right lower limbs. However, this can be accounted for by the fact that the IT is an attachment site for the iliofemoral ligament (Standring, 2008), not a site of muscle attachment, and studies show that entheseal morphology is affected by age and sex, rather than by limb dominance (Milella et al., 2012).

The data show that there was no statistical significance between the IT coordinates on the $\mathrm{x}$ or $\mathrm{y}$ axis and sex $(P>0.05)$. This adds to, and is supportive of, data reported by Kielbasinski Podlaszewska et al. (2017) that showed that there was no difference between IT prominence and sex. The correlation coefficients were all below -0.3, showing negligible correlation (Mukaka, 2012). 
The aim of this study was to provide further evidence to either refute or support the notion that the IT is a reliable landmark or "lighthouse" (Williams et al., 2015) for making the femoral neck cut during DAA hip arthroplasties. Our results show that the position of the IT is not affected by sex or femoral side and can therefore be considered a consistent and reliable reference point. The $19^{\text {th }}$ century skeletal population at St Bride's has previously been used to test the reliability of modern methods of sex determination such as the expression of sexual dimorphism in the foramen magnum (Gapert et al., 2009). Their study concluded that modern techniques of sex determination used in the forensic field today were equally valid in the St Bride's population, suggesting that skeletal elements are comparable to a modern-day population.

Limitations

While forensic analysis suggests that the St Bride's skeletons are comparable to a modern population, post-mortem and taphonomic damage may have affected the femurs in this study. Ideally, the study should be repeated using a modern-day skeletal assemblage with known demography.

\section{Conclusion}

This osteological study shows that $t$ there was no significant association between the position of the IT with sex or side, nor did the position of the IT vary significantly with femoral neck dimensions. Therefore, the IT can be confirmed as a consistent and reliable landmark for surgeons during DAA hip replacement. It is hoped that the results from this study may be of use to orthopaedic surgeons and research osteologists. 


\section{References}

Anakwenze, O. A., Khanna, K., Levine, W.N., Ahmad, C.S. (2014). Characterization of the supinator tubercle for lateral ulnar collateral ligament reconstruction. Orthop J Sports Med, 2(4), 2325967114530969 doi: $10.1177 / 2325967114530969$.

Chamay, A. and Tschantz, A. (1971). Mechanical influences in bone remodelling. Experimental research on Wolff's law. J Biomech, 5(2), 173-180.

Connolly, K. P. and Kamath, A. F. (2016). Direct anterior total hip arthroplasty: comparative outcomes and contemporary results. World J Orthop, 7(2), 94 doi: 10.5312/wjo.v7.i2.94.

Fahmy, M., Williams, R.L., Adds, P. (2018). The innominate tubercle of the femur: a pilot study of its position in relation to the neck of the femur. Clin Anat, 31(8), E40-E60.

Frazer, J. (1920). The Anatomy of The Human Skeleton, Second Edition. London: J and A Churchill. pp 138-140.

Genge, N. (2004). The Forensic Casebook: The Science Of Crime Scene Investigation. London: Ebury Press.

Glassman, D. M. and Dana, S. E. (1992). Handedness and the bilateral asymmetry of the jugular foramen. J Forensic Sci, 37(1), 140-146.

Gray, H. (1918). Anatomy of the Human Body, Twentieth Edition. Philadelphia: Lea \& Febiger. pp 244245.

Kanchan, T., Kumar, M., Kumar, P. (2007). Handedness in skeletal remains. J Indian Acad Forensic Med, 29(1), 42-43.

Keggi, J. M. (2014). The direct anterior approach: here today, gone tomorrow-Opposes. Semin Arthroplasty, 25(2), 127-129. doi: 10.1053/j.sart.2014.04.007.

Kielbasinski Podlaszewska, O., Bekvalac, J., Williams, R.L., Adds, P.J. (2017). The innominate tubercle of the femur: Application to anterior surgical approaches to the hip. Clin Anat, 30(5), 578-584. doi: 10.1002/ca.22870.

Knüsel C (2000). Bone adaptation and its relationship to physical activity in the past. In: Cox, M and 
Mays, S. (Eds.) Human Osteology: In Archaeology and Forensic Science. London: Greenwich Medical Media. pp 381-402.

Makhni, E. C., Khanna, K., Simpson, M.T., Redler, L.H., Anakwenze, O.A., Li, R., Ahmad, C.S. (2014). Medial epicondyle morphology in elite overhead athletes: a closer look using 3-dimensional computer simulation. Orthop J Sports Med, 2(1), 2325967113517211 doi: 10.1177/2325967113517211.

Meeusen, R. A., Christensen A. M., Hefner, J. T. The use of femoral neck axis length to estimate sex and ancestry (2015). J Forensic Sci, 60(5), 1300-1304. doi: 10.1111/1556-4029.12820

Milella, M., Giovanna Belcastro, M., Zollikofer, C.P., Mariotti, V. (2012). The effect of age, sex, and physical activity on entheseal morphology in a contemporary Italian skeletal collection. Am J Phys Anthropol, 148(3), 379-388. doi: 10.1002/ajpa.22060.

Mukaka, M. M. (2012). Statistics corner: A guide to appropriate use of correlation coefficient in medical research. Malawi Med J, 24(3), 69-71.

Museum of London (2015). St Bride's Church Fleet Street. Available at: https://www.museumoflondon.org.uk/collections/other-collection-databases-and-libraries/centrehuman-bioarchaeology/osteological-database/post-medieval-cemeteries/st-brides-church-fleetstreet. [Accessed 10 May 2020].

Museum of London. (2018). Wellcome Osteological Research Database. Available at: https://www.museumoflondon.org.uk/collections/other-collection-databases-and-libraries/centrehuman-bioarchaeology/osteological-database. [Accessed: 9th January 2018].

O’Rahilly, R., Müller, F., Carpenter, S., Swenson, R. (2004). Basic Human Anatomy. Available at: https://www.dartmouth.edu/ humananatomy/index.html (Accessed: 12 April 2020).

Prasad, R., Vettivel, S., Isaac, B., Jeyaseelan, L. and Chandi, G. (1996) Angle of torsion of the femur and its correlates. Clin Anat, 9(2), 109-117 doi: 10.1002/(SICI)1098-2353(1996)9:2<109::AIDCA3>3.0.CO;2-C.

Standring, S. (2008). Gray's Anatomy: The Anatomical Basis of Clinical Practice, 40th Edition. Edinburgh: Elsevier. 
Thomas, A., Bajada, S., Antonios, T., Bekvalac, J., Adds, P., Williams, R. (2018). Reliability evaluation of inter-eminence line, Akagi and Dalury lines for intraoperative tibial rotation: an osteology-based study. Knee, 25 (3,) 453-458 DOI: https://doi.org/10.1016/j.knee.2018.02.010.

Williams, R., Goodson, M., Danayana, K., Maheson, M. (2015). The innominate tubercle: A lighthouse for the femoral neck cut during the direct anterior approach. Clin Anat, 28(3), 419. doi: 10.1002/ca.22508.

Williams, R., Goodson, M., Maheson, M. (2014). The innominate tubercle and superimposition of the rasp - an accurate technique for the femoral neck osteotomy during the direct anterior approach to the hip. [Poster Presentation] European Hip Society, Stockholm. 


\section{FIGURE LEGENDS}

Figure 1. The innominate tubercle of the femur (circled)

Figure 2. Coordinates of the innominate tubercle (marked with white tac). Black lines, reference grid lines; white lines, $x$ and $y$ axes; dotted lines, $x$ and $y$ coordinates of the tubercle.

Figure 3a. Contour plot of IT position in males. Contours indicate the relative probability of a value falling within that area. Probability indicated on a scale from red (most dense) to light green (least dense)

Figure 3b. Contour plot of IT position in females. Contours indicate the relative probability of a value falling within that area. Probability indicated on a scale from red (most dense) to light green (least dense)

Figure 3c. Contour plot of IT position in males and females combined. Contours indicate the relative probability of a value falling within that area. Probability indicated on a scale from red (most dense) to light green (least dense) 


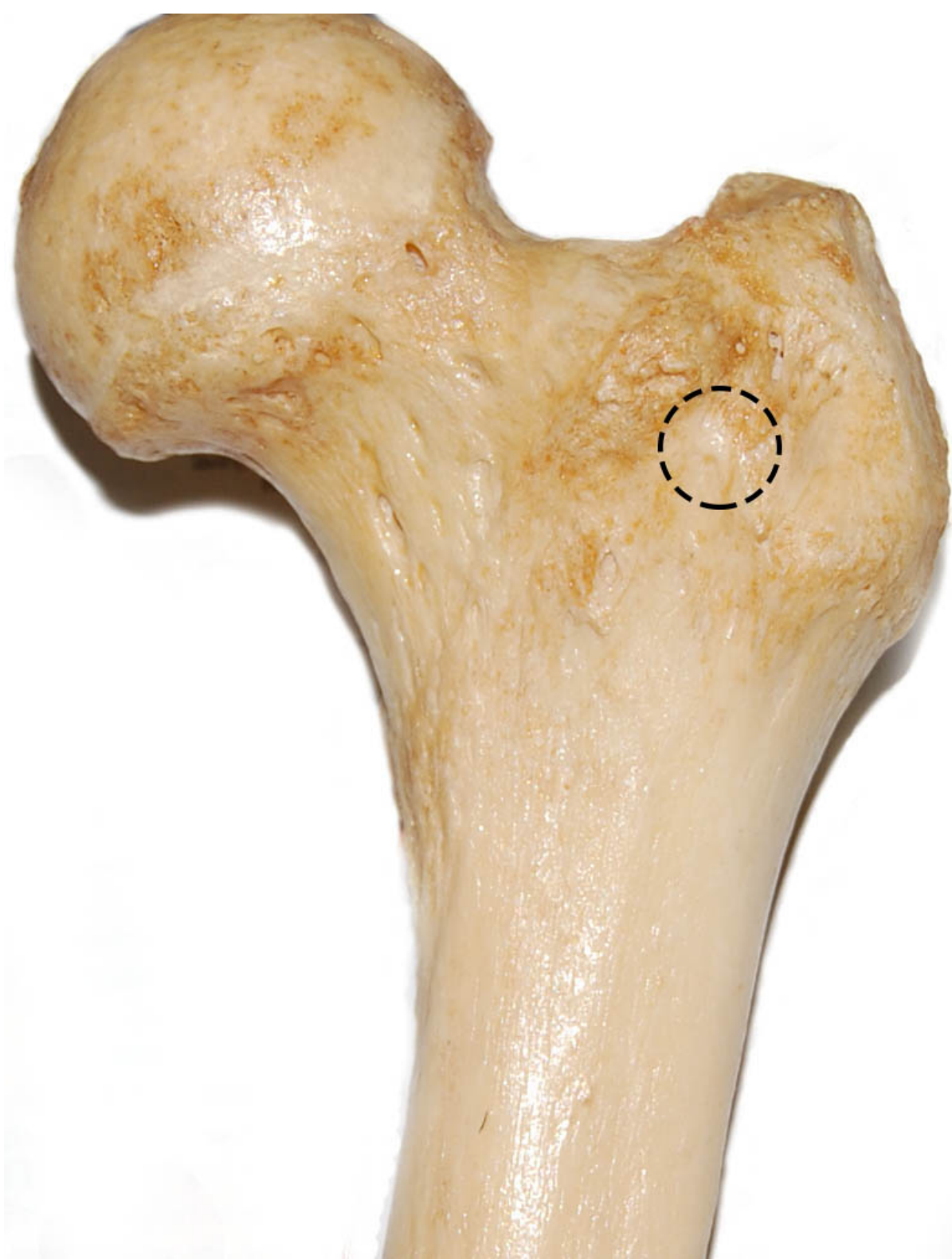

Figure 1. Figure 1. The innominate tubercle of the femur (circled)

John Wiley and Sons, Inc. 


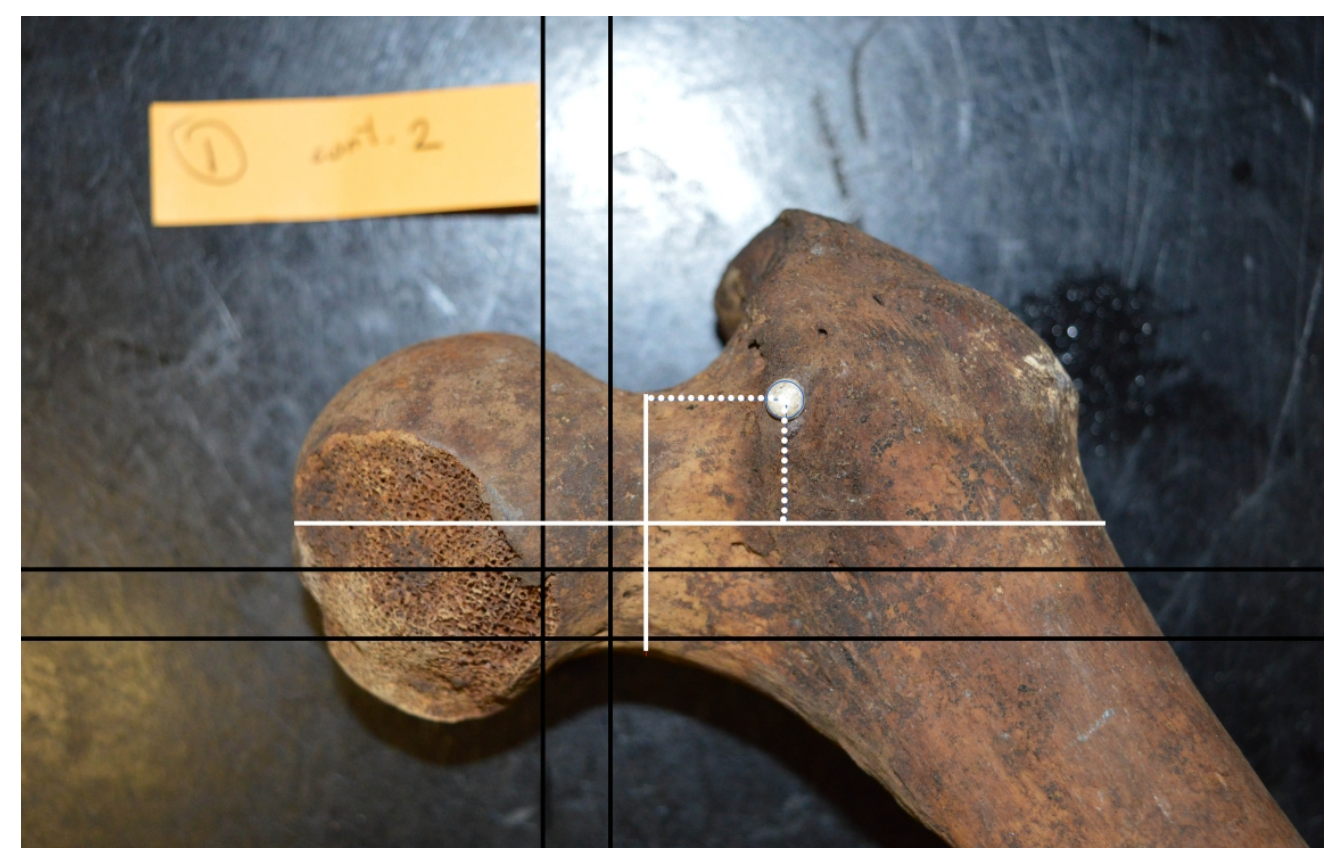

Figure 2. Coordinates of the innominate tubercle (marked with white tac). Black lines, reference grid lines; white lines, $x$ and $y$ axes; dotted lines, $x$ and $y$ coordinates of the tubercle. 


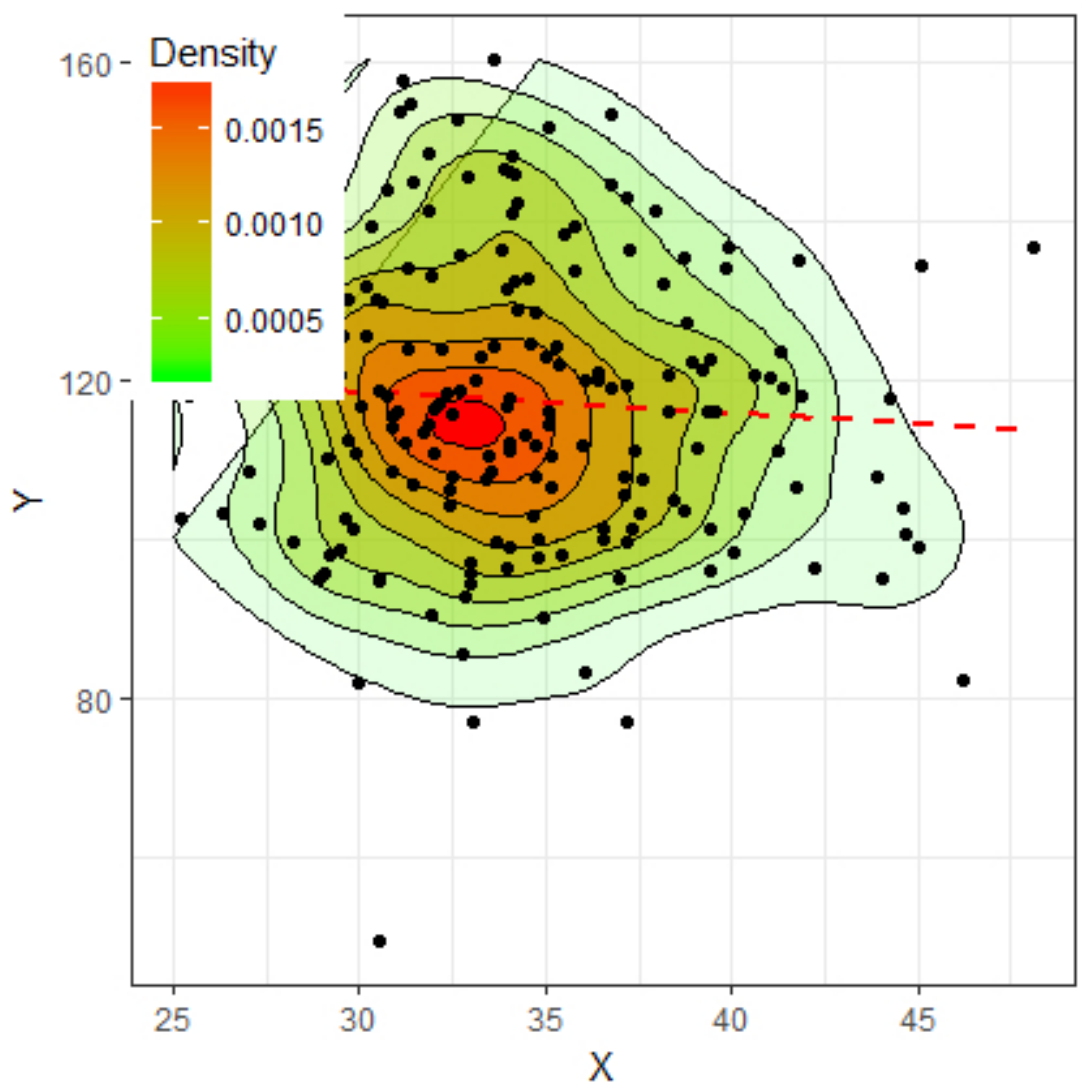

Figure 3a. Contour plot of IT position in males. Contours indicate the relative probability of a value falling within that area. Probability indicated on a scale from red (most dense) to light green (least dense) 


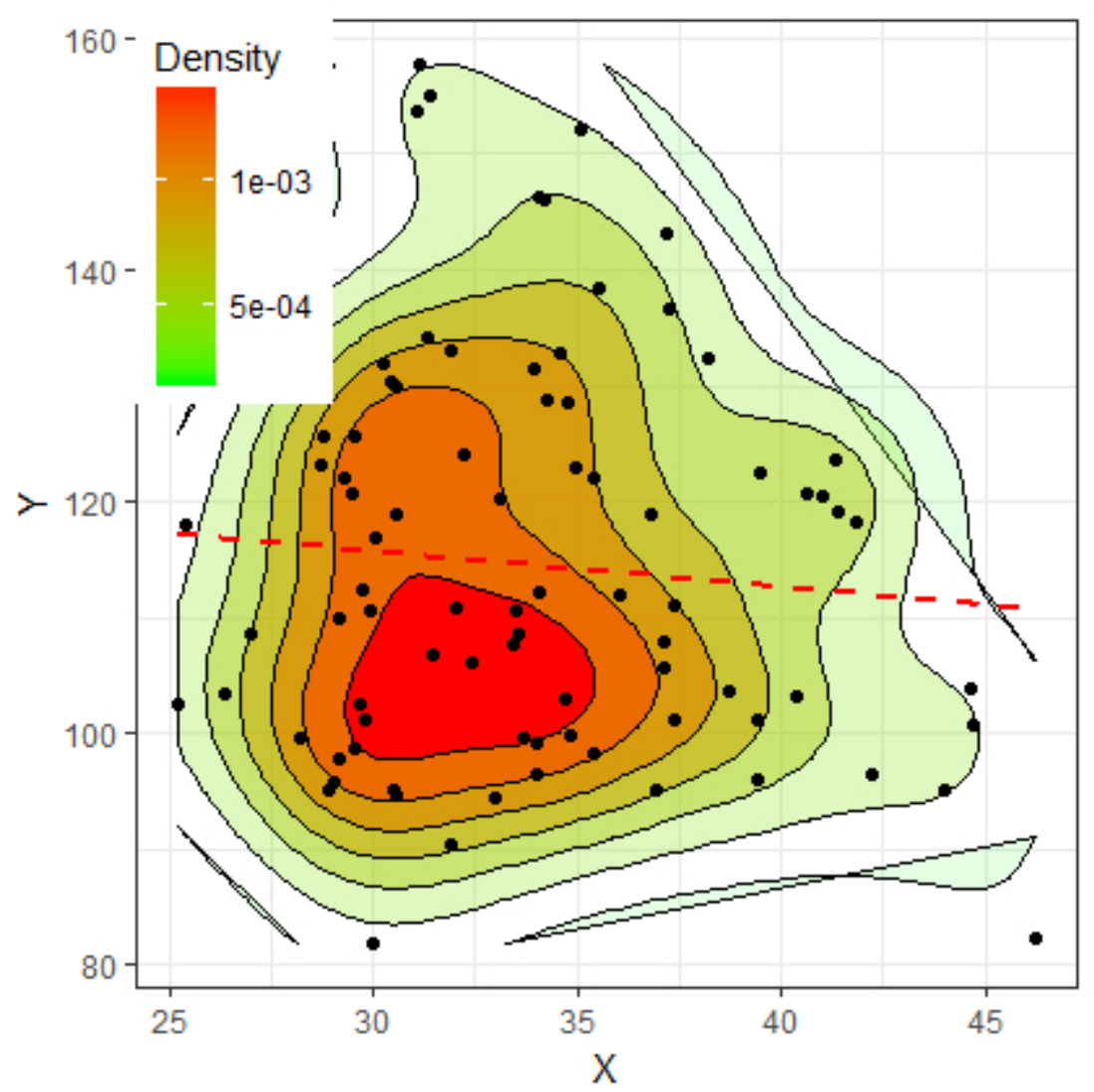

Figure 3b. Contour plot of IT position in females. Contours indicate the relative probability of a value falling within that area. Probability indicated on a scale from red (most dense) to light green (least dense) 


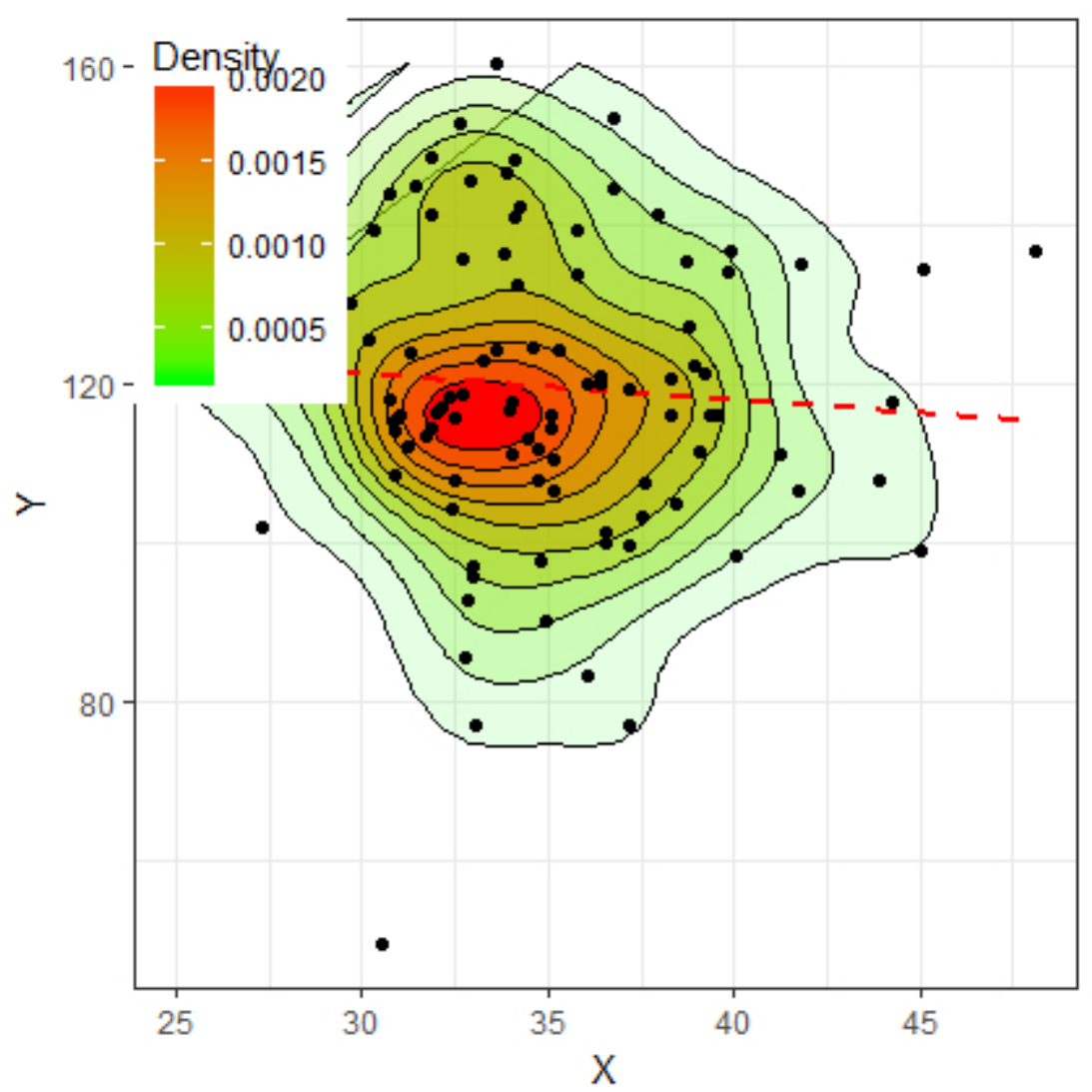

Figure 3c. Contour plot of IT position in males and females combined. Contours indicate the relative probability of a value falling within that area. Probability indicated on a scale from red (most dense) to light green (least dense) 